9. Teoreticheskie osnovy radiolokacii / Shirman Ya. D., Golikov V. N., Busygin I. N., Kostin G. A., Manzhos V. N., Minervin N. N. et. al. Moscow: Sovetskoe radio, $1970.560 \mathrm{p}$.

10. PinPoint: An asynchronous time-based location determination system / Youssef M., Youssef A., Rieger C., Shankar U., Agrawala A. // Proceedings of the 4th international conference on Mobile systems, applications and services - MobiSys 2006. 2006. P. 165-176. doi: https://doi.org/10.1145/1134680.1134698

11. Cong L., Zhuang W. Hybrid TDOA/AOA mobile user location for wideband CDMA cellular systems // IEEE Transactions on Wireless Communications. 2002. Vol. 1, Issue 3. P. 439-447. doi: https://doi.org/10.1109/twc.2002.800542

12. Bargshady N., Garza G., Pahlavan K. Precise Tracking of Things via Hybrid 3-D Fingerprint Database and Kernel Method Particle Filter // IEEE Sensors Journal. 2016. Vol. 16, Issue 24. P. 8963-8971. doi: https://doi.org/10.1109/jsen.2016.2616758

13. Atia M. M., Noureldin A., Korenberg M. J. Dynamic Propagation Modeling for Mobile Users' Position and Heading Estimation in Wireless Local Area Networks // IEEE Wireless Communications Letters. 2012. Vol. 1, Issue 2. P. 101-104. doi: https://doi.org/ 10.1109/wcl.2012.020612.110279

14. ITU-R P.1238-9 - Propagation data and prediction methods for the planning of indoor radio communication systems and the radio local area networks in the frequency range $300 \mathrm{MHz}$ to $100 \mathrm{GHz}$. Geneva: ITU-R Recommendations, 2017.

15. Zymbler M. L., Miniakhmetov R. M., Rogov A. A. The survey of indoor positioning algorithms for mobile devices // Bulletin of the South Ural State University. Series «Computational Mathematics and Software Engineering». 2013. Vol. 2, Issue 2. P. 83-96. doi: https://doi.org/10.14529/cmse130207

\begin{tabular}{|c|}
\hline 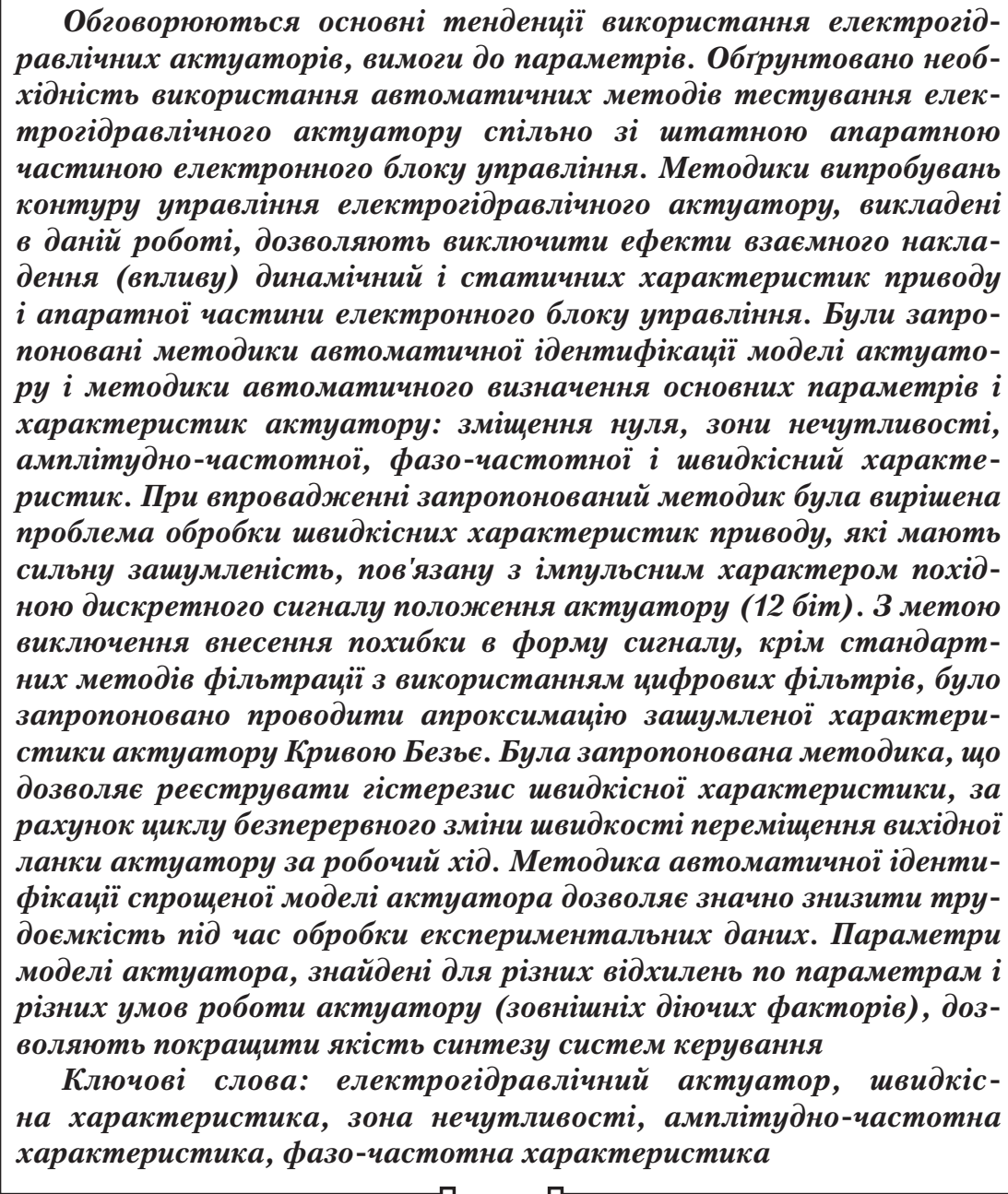 \\
\hline
\end{tabular}

UDC 629.735

DOI: $10.15587 / 1729-4061.2019 .154837$

\section{DEVELOPMENT OF PROCEDURES FOR DETERMINING THE PARAMETERS OF AN AIRCRAFT SERVO ACTUATOR}

\author{
E. Kon on yk hin \\ Head of Research Department \\ PrJSC FED \\ Symska str., 132, Kharkiv, \\ Ukraine, 61000 \\ E-mail: ekononykhin@icloud.com
}

\section{Introduction}

Electrohydraulic servo actuators (EHSA) are most widely used in systems of automatic control of aircraft engines for moving mechanization elements and in flight control systems for controlling steering surfaces [1, 2]. Liquid taken from a high-pressure fuel pump in automatic control systems of air- craft engines (ACS AE) or from hydraulic pumps of the flight control systems is the working fluid for an electro-hydraulic actuator. Upon electrical commands, electrohydraulic amplifier (EHA) which is a part of the actuator converts low-power control signals into proportional hydraulic commands of considerable power. Upon distribution, working fluid enters hydraulic cylinder (to servo piston) and forms mechanical command. 
Currently, when conducting EHSA tests, a limited number of actuator parameters are conventionally controlled according to outdated procedures adapted for universal test equipment with a low degree of automation [3]. Such tests do not permit to fully control stability of static and dynamic characteristics of the actuator when operating in a control loop with modern digital regulators [4-6].

Control algorithms interact with the EHSA through hardware of the electronic control unit possessing certain dynamic and static characteristics which should also be considered when developing dynamic models of control objects. Use of hardware and digital filters in the structure of electronic control units of aviation actuators results in 1.5-6 times reduction of the control object pass band.

Relevance of this study is explained by necessity of development of automatic methods for EHSA testing which would take into account influence of hardware in electronic control units. Also, solution of the problem of building up volumes of information obtained in tests which would improve quality of synthesis of control algorithms and make better control of stability of actuator characteristics is urgent.

\section{Literature review and problem statement}

When developing state-of-the-art digital regulators, problems of ensuring minimum time of transient processes and maximum stability margin of regulators are solved [7]. Often, in order to ensure maximum dynamic parameters of the control loop, a regulator is adjusted close to the oscillatory stability boundary. Therefore, oscillations can occur because of a considerable spread of parameters of the actuator control loop. Improvement of the control loop parameters can be achieved using adaptive algorithms. However, regulator complexity greatly increases while risk of auto-oscillations remains [8]. Solution of the problem of improving dynamic qualities of the control loop while maintaining sufficient stability margins is possible due to application of mathematical models of the actuator with a high degree of compliance of its characteristics with the actual control object $[9,10]$.

A model and methods for identifying an electropneumatic clutch actuator was proposed in [11]. However, this model does not take into account nonlinearity and dynamic and static characteristics of an electropneumatic (electrohydraulic) converter. It was shown in [12] that the main impact on characteristics of the actuator in general is exerted by characteristics of an electro-hydraulic converter and, therefore, application of model [11] will lead to significant errors. Masses of the piston and working fluid considered in model [11] are negligible for most electro-hydraulic actuators compared to the force and the mass attached at the output link.

Necessity of application of a mathematical model of a hydraulic valve enabling reproduction of nonlinearities to improve quality of the actuator control loop was demonstrated in [13] and methods for determining hysteresis of flow characteristics of a hydraulic valve are described in [14]. The models given in [13] and [14] do not take into account the dynamics and non-linearity of speed characteristic of an electro-hydraulic amplifier, and the model of hysteresis of the speed characteristic does not take into account hysteresis of displacement of the sliding piston-type selector valve relative to the control signal. Also, known are models of electro-hydraulic converters of a high level of detail [15, 16], however, identification of these models is difficult because of a large number of parameters.

Thus, the known studies do not contain description of a mathematical model and methods for determining actuator parameters that would fully correspond to characteristics of aviation servo actuators. The task of developing a mathematical model of an electro-hydraulic actuator which would make it possible to reproduce main characteristics with a sufficient accuracy is pressing. It is also necessary that such a model have the simplest structure to simplify the task of determining the model parameters.

Determination of the model parameters using standard general-purpose software, for example, MatLab System Identification Toolbox, is difficult because of complexity of integration with the actuator control algorithms used in the structure of standard electronic control units. In the case of using general purpose software for model identification, the procedure for preparing initial data becomes much more complicated and qualification requirements to the test bench operators get stricter.

Despite the high information content, control of parameters of the mathematical actuator model at the stage of acceptance tests was not widespread. This is because requirements to basic parameters defined in EHSA standards are mainly used in technical specifications for EHSA development [5]. The main parameters and characteristics of EHSA include zero shift, dead space, amplitude-frequency, phase-frequency and speed characteristics. Despite obvious advantages, methods for automatic determination of main parameters and characteristics of EHSA were poorly studied in the known literature.

\section{The aim and objectives of the study}

The study objective was to develop procedures for automatic determining parameters of aviation servo-actuator control loop. Procedures must enable taking into account static and dynamic characteristics of hardware of the electronic control unit (in connection with which, automated testing algorithms must function as a part of a regular electronic unit).

To achieve this objective, the following tasks were set:

- develop a mathematical model of an electro-hydraulic actuator which would make it possible to reproduce with sufficient accuracy main characteristics of the actuator and have the simplest structure to make easier the task of parameter determination;

- develop procedures for automatic experimental determination of parameters of the mathematical actuator model together with hardware of the electronic control unit;

- develop procedures for automatic experimental determination of main parameters and characteristics of the actuator

\section{Mathematical model of an electro-hydraulic actuator}

Block diagram of the proposed mathematical model of an electro-hydraulic actuator is shown in Fig. 1. Current command to the EHA, $I_{i n}$, is the input parameter of the mathematical model and the output link movement speed, $V$, is its output. Blocks $a$ and $b$ simulate dynamics and hysteresis of speed characteristic of the actuator, respectively, 
according to the control signal. Block $a$ can be described by the equation:

$$
T \frac{d^{2} I_{T}}{d t^{2}}+I_{T}=I_{i n}
$$

where $I_{T}$ is the control signal at the output of the block $a$; $T$ is the time constant of the system.

The hysteresis model (block $b$ ) can be described by the following dependence:

$$
\begin{aligned}
& I_{T}>I_{B L i-1}+B \rightarrow I_{B L}=I_{T}-B, I_{B L i-1}=I_{B L} ; \\
& I_{T}<I_{B L i-1}-B \rightarrow I_{B L}=I_{T}+B, I_{B L i-1}=I_{B L} ; \\
& I_{B L i-1}-B<I_{T}<I_{B L i-1}+B \rightarrow I_{B L}=I_{B L i-1},
\end{aligned}
$$

where $I_{B L}$ is the control signal at the output of the block $b$; $B$ is the dead space threshold.

To describe dependence of speed of movement of the output link on the control signal $V_{0}=f\left(I_{B L}\right)$ (block $b$ ) in absence of loads and friction forces, a tabular form of recording with approximation by a cubic spline or lines was used.

The effect of load at the output link on speed characteristics of the actuator is described by the dependences shown in block $d$ in Fig. 1.
Thus, mathematical model of the EHSA control loop (its structure is shown in Fig. 1) allows one to establish relationship between the control signal $I_{\text {in }}$ and the actuator movement speed $V$ taking into account the output link load $F$.

\section{Procedures for determining the EHSA parameters}

An electronic unit connected to a personal computer was used in the test bench for automatic determination of the EHSA loop parameters. The electronic unit consisted of three main modules: a module for the test bench control, a module for analysis of signals from sensors and hardware identical to the standard EHSA control units. The hardware identical to the standard control unit performs function of a digital-to-analog and analog-to-digital converter for control and controlled signals (from input and output) of the EHSA. Analysis of the control and controlled signals makes it is possible to determine main parameters of the actuator: zero shift, dead space, amplitude-frequency, phase-frequency and speed characteristics of the actuator.

Parameters of the blocks $a, b$ and $c$ are determined from experimental data in automatic determination of the zero shift, dead space, amplitude-frequency, phase-frequency and speed characteristic of the actuator. Parameters of the block $d$ are determined using additional electronic pressure sensors in cavities of the hydraulic cylinder connected to the test bench equipment independent of the standard hardware of the electronic unit.

The software for automatic determination of the EHSA loop parameters consisted of lower-level software embedded in the electronic control unit and upper-level software in a personal

Fig. 1. Block diagram of a mathematical model of an electro-hydraulic actuator: transfer function (a); hysteresis $(b)$; speed characteristic of the actuator $(c)$, hydraulic cylinder model $(d)$

Maximum force developed by the actuator depends on the piston area $S_{p}$ and nominal pressure $P_{s}$. at the inlet to the electrohydraulic amplifier. Steepness of change of the pressure differential in cavities of the hydraulic cylinder when the control signal changes relative to the zero-shift current $I_{0}$ is described in the mathematical model by coefficient $k_{p}$. Friction forces in the hydraulic cylinder are simulated by the pressure differential $\Delta P_{f r}$ between cavities of the hydraulic cylinder necessary to overcome friction forces.

The zero shift $I_{0}$ (block $d$ ) is determined from the actuator speed characteristic (block $c$ ) as a current command at a zero speed. The piston area $S_{\mathrm{p}}$ and pressure $P_{\mathrm{s}}$ are determined by the design and operating conditions of the actuator. Steepness of change of the pressure differential in the hydraulic cylinder cavities, $k_{\mathrm{p}}$, according to the control signal, $I$, depends on geometrical parameters of the "sliding valve-sleeve" pair of the EGA. The pressure differential in cavities of the hydraulic cylinder necessary to overcome friction forces, $k_{f r}$, depends on parameters of seals and piston supports.

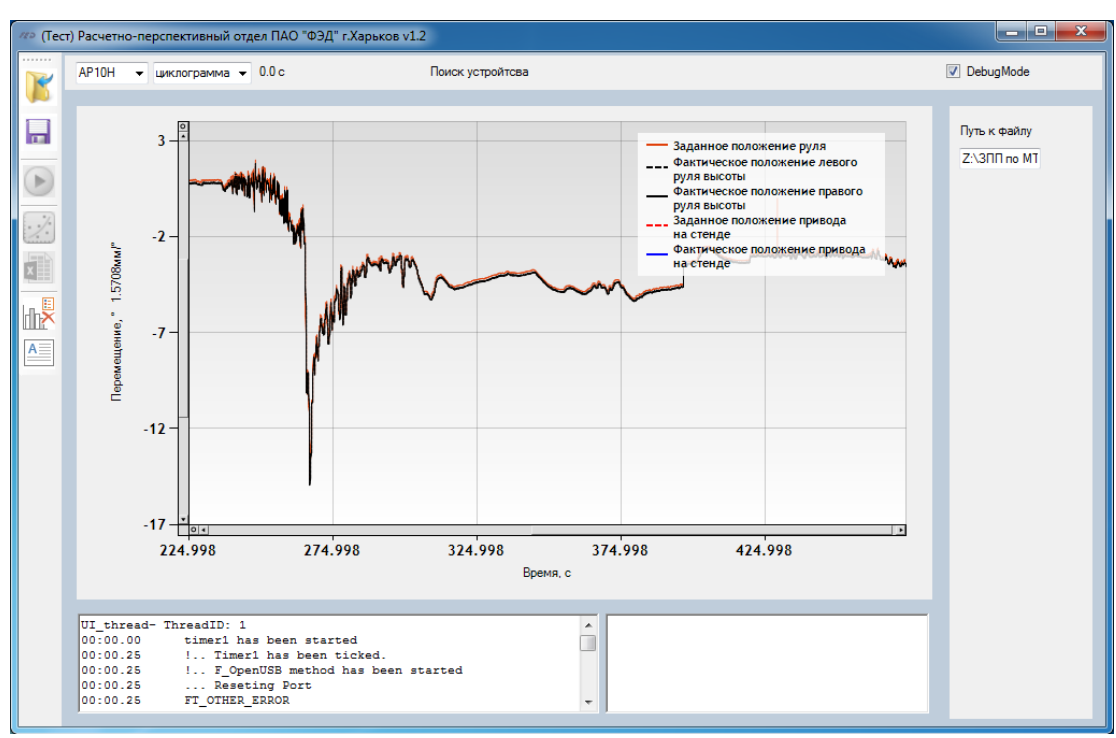

Fig. 2. Central window of software for reading and analysis of characteristics of the actuator and the actuator control loop computer (Fig. 2). The lower-level software was responsible for generating control signals and processing signals from sensors according to the algorithms corresponding to each test type. The upper-level software was responsible for acquisition, postprocessing and visualization of experimental data 
Characteristics and parameters of the actuator used hereinafter as an example were obtained in the process of automatic determination of aviation servo-actuator parameters.

\section{1. Procedure for automatic determination of static} parameters of the actuator and the model

Static parameters of the actuator can include dead space and shift of the actuator dead space. Static parameters of the model can include $k_{p}, \Delta P_{f r}$ and hysteresis. Existing methods for determining the dead space [18] do not ensure separate analysis of hysteresis of the speed characteristic and influence of friction forces on the output link. Since the dead space depends on hysteresis of the speed characteristic and friction forces in the hydraulic cylinder (Fig. 1), it is advisable to combine these types of checks. As it was said, in order to determine parameters $k_{p}$ and $\Delta P_{f r}$, pressure sensors are attached to the hydraulic cylinder cavities. The hydraulic cylinder signal is automatically logged by the bench. To log the rest of parameters and characteristics, it is enough to use hardware of the regular control unit.

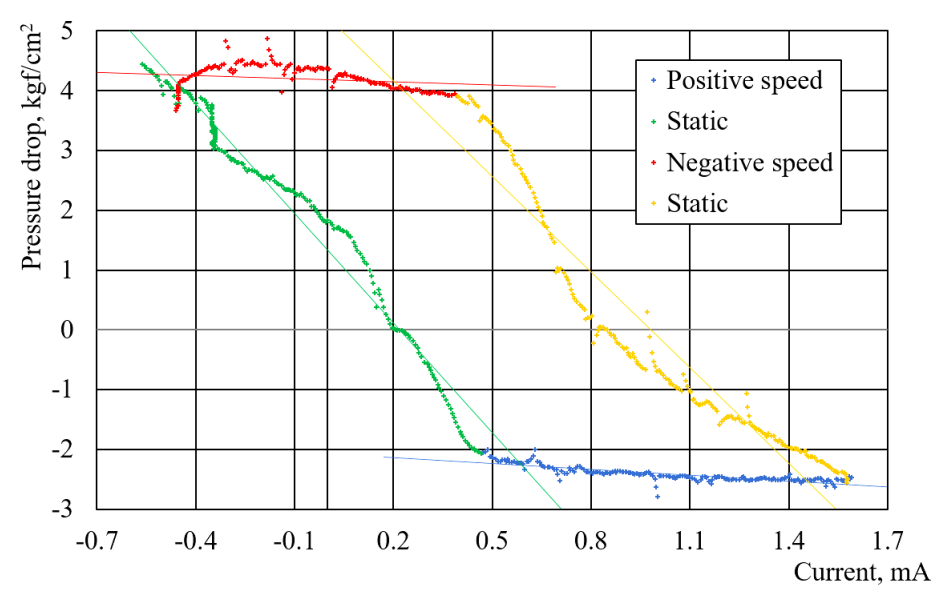

Fig. 3. EHSA hysteresis by the cavity pressure differential
To determine parameters $k_{\mathrm{p}}$ and $\Delta P_{f r}$ and hysteresis, a saw-toothed cyclic signal of the set actuator position is formed. The control signal generates a regulator with the control signal offset to a predefined dead space zone. The control signal, position of the output link and pressure in the hydraulic cylinder cavities are recorded during the retraction-extension actuator cycle. Then, the 'position - control signal' and 'pressure differential - control signal' point fields are analyzed with their division into three groups: a static output link, an output link moved in a positive direction and an output link moved in the negative direction (Fig. 3). Parameters $k_{p}$ and $\Delta P_{f r}$ and hysteresis are determined for several ranges of positions of the actuator output link with presetting the output actuator link to lower and upper boundaries of each of the ranges.

Zero shift $I_{0}$ and the dead space of the actuator are determined from the values of the control signals necessary to start movement of the actuator in different directions (Fig. 2). Steepness of change of the pressure differential, $k_{p}$, in cavities of the hydraulic cylinder is defined as the angle of inclination of the graphs of the pressure differential in cavities of the hydraulic cylinder at a static output link (Fig. 2). The pressure differential, $\Delta P_{\mathrm{fr}}$, in the cavities of the hydraulic cylinder required to overcome friction forces is determined by shift of graphs of the pressure differential when the output link is static (Fig. 2).

Hysteresis B (Fig. 1) is determined as a difference between the dead space and the $\Delta P_{f r} / k_{p}$ ratio.

5. 2. Procedure for automatic determination of the amplitude-frequency and phase-frequency characteristics and dynamic parameters of the model

To determine the amplitude-frequency and phase-frequency characteristics and dynamic parameters of the model, experimental data on position of the actuator output link when applying a sinusoidal control signal were used. Dynamic characteristics were determined for preset frequencies and amplitudes of control signals (Fig. 4).

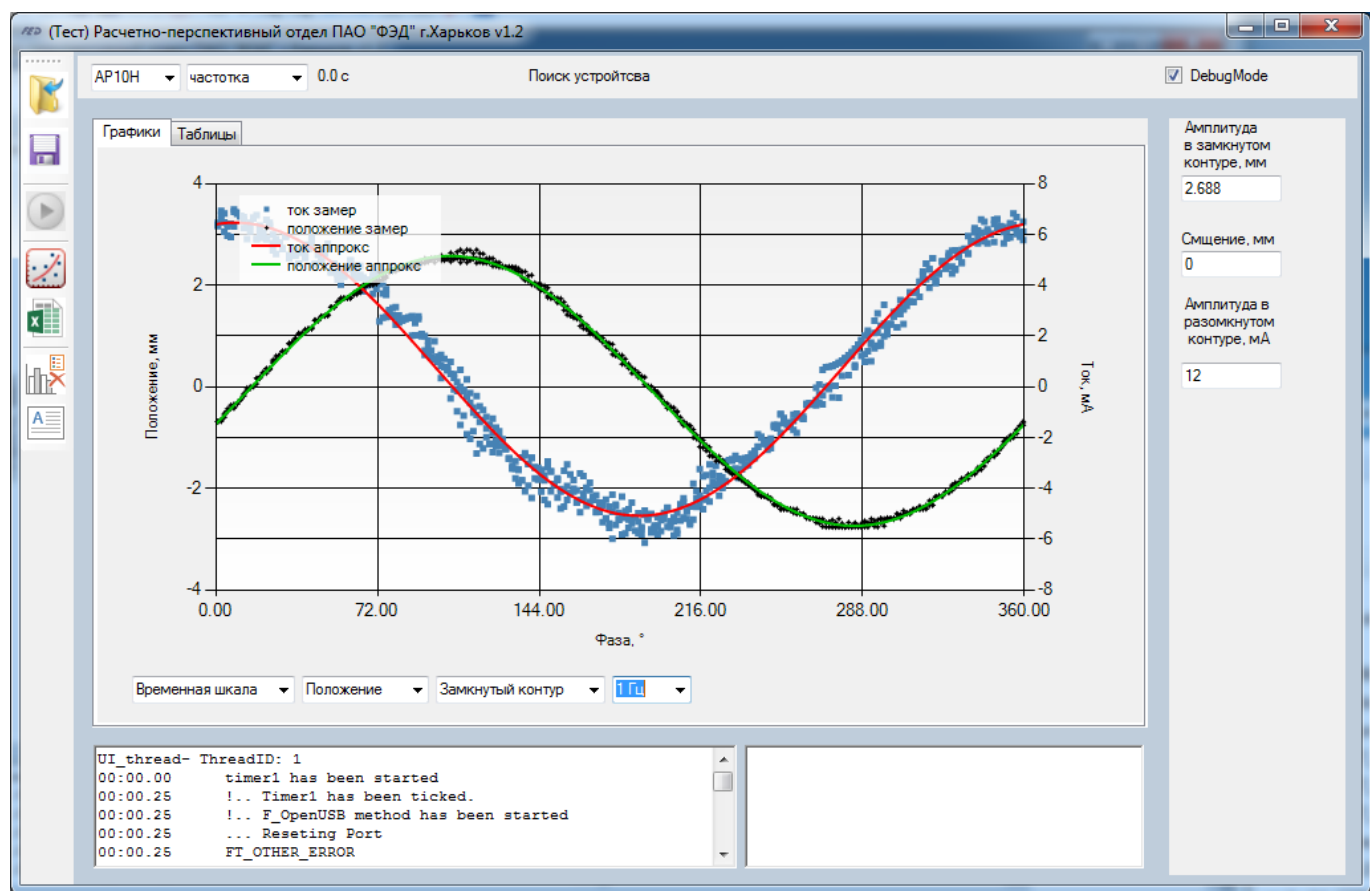

Fig. 4. Software interface when reading the amplitude-frequency and phase-frequency characteristics 
Position of the actuator was recorded during the oscillation cycle for each of the set frequencies and amplitudes of the control signal. To prevent the actuator from shifting during the oscillation cycle, current position of the actuator was compared with the position measured in the previous oscillation period. Based on information about the change of the actuator position in the oscillation cycle, an additional component of the control signal was formed using an integrated controller. This compensation method prevents occurrence of additional harmonics in the control signal.

Since the mathematical model of the actuator (Fig. 1) has nonlinearities in its structure, it is advisable to determine the time constant $T$ using the gradient descent method $[19,20]$. Root-mean-square deviation of the actuator position determined by the mathematical model and obtained in the experiment was taken as the target function.

As amplitudes of the output signal changed at different frequencies, the root-mean-square deviations before summation over all frequencies were normalized by the amplitude of the output signal.

To determine amplitude-frequency and phase-frequency characteristics, the obtained data were approximated by an ideal sinusoidal signal in the oscillation period for each of the frequencies (Fig. 3). The ideal sinusoidal signal is described by the dependence:

$$
x_{a p r}=a_{1}+a_{2} \cdot \sin \left(\alpha+a_{3}\right) \text {, }
$$

where $x_{\text {apr }}$ is the actuator position based on the results of approximation; $\alpha$ is the control signal phase in relation to which the phase lag $a_{3}$ of the actuator position is determined; $a_{1}$ is the actuator position in the vicinity of which dynamic parameters are measured; $a_{2}$ is the actuator movement amplitude.

Approximation was carried out with minimization of the root-mean-square deviation between measured, $x_{\text {meas }}$, and approximated, $x_{\mathrm{app}}$, actuator positions:

$$
\begin{aligned}
& \sigma=\sum_{i=0}^{n} \sigma_{i} ; \\
& \sigma_{i}=\left(x_{\text {meas } i}-x_{a p r}\left(\alpha_{i}\right)\right)^{2} .
\end{aligned}
$$

Approximation was carried out by an iterative method [20]. As a result of this approximation, values of $a_{1}, a_{2}$ and $a_{3}$ were determined. The vector of square deviations, $R$, and the matrix of derivatives of square deviations, $J$, for each of the sought values for all points of the measured position were formed at each step:

$$
J=\left(\begin{array}{lll}
\frac{\partial \sigma_{1}}{\partial a_{1}} & \frac{\partial \sigma_{1}}{\partial a_{2}} & \frac{\partial \sigma_{1}}{\partial a_{3}} \\
\frac{\partial \sigma_{2}}{\partial a_{1}} & \frac{\partial \sigma_{2}}{\partial a_{2}} & \frac{\partial \sigma_{2}}{\partial a_{3}} \\
\cdots & & \\
\frac{\partial \sigma_{n}}{\partial a_{1}} & \frac{\partial \sigma_{n}}{\partial a_{2}} & \frac{\partial \sigma_{n}}{\partial a_{3}}
\end{array}\right), \quad R=\left(\begin{array}{c}
\sigma_{1} \\
\sigma_{1} \\
\ldots \\
\sigma_{n} \\
\end{array}\right),
$$

where partial derivatives are calculated by the following dependences:

$$
\frac{\partial \sigma_{i}}{\partial a_{1}}=2 a_{1}-2 x_{\text {meas }}+2 a_{2} \cdot \sin \left(\alpha+a_{3}\right),
$$

$$
\begin{aligned}
& \frac{\partial \sigma_{i}}{\partial a_{2}}=2 \cdot \sin \left(\alpha+a_{3}\right) \cdot\left(a_{1}-x_{\text {meas }}+a_{2} \cdot \sin \left(\alpha+a_{3}\right)\right), \\
& \frac{\partial \sigma_{i}}{\partial a_{3}}=2 a_{2} \cdot \cos \left(\alpha+a_{3}\right) \cdot\left(a_{1}-x_{\text {meas }}+a_{2} \cdot \sin \left(\alpha+a_{3}\right)\right) .
\end{aligned}
$$

Next, the vector of deviations was calculated from the sought values:

$$
\Delta=R J\left(J^{T} J\right)^{-1} .
$$

The vector $\Delta$ components are the input for the PID regulators. The sought values of $a_{1}, a_{2}$ and $a_{3}$ are formed at the regulator output. For $a_{3}$, condition of a shift by $2 \pi$ was additionally introduced in the direction of increase or decrease in the component in the case of overrunning the boundaries from 0 to $2 \pi$, respectively. As the rms value of the vector $\Delta$ components of a certain threshold value decreases, exit from the iterative process takes place.

Following determination of the phase lag, $a_{3}$, and the amplitude lag, $a_{2}$, amplitude-frequency and phase-frequency characteristics are built automatically.

\section{3. Procedure for automatic determination of the} actuator speed characteristic

To determine the actuator speed characteristic in absence of an external load $V_{0}=f(I)$, two methods were proposed: for a continuous change of the control signal during the working stroke and for fixed levels of the control signals.

When the method for determining speed characteristic for the $n$-th number of fixed levels of the control signal is used, one can determine speed change during the working stroke in addition to the average speed of the output link movement. Based on the dead space information for the control signal determined according to the procedure of hysteresis determination described above, the control signal values are automatically found to determine speed of the output link movement in both directions. Next, the control signals of fixed levels are successively applied $n$ times to move the actuator in both directions. Average speeds for each of the levels of the control signal are determined using the measured speed of the output link movement (Fig. 5).

With the method of determining the speed characteristic at a variable change of the control signal per working stroke, it is possible to record the speed characteristic taking into account hysteresis and the dead space due to the presence of sections of both increase and decrease in the control signal. This method is suitable for determining the speed characteristic of the actuators in which speed of the output link movement does not depend on position. Before measuring the speed characteristic, the actuator is set to the position corresponding to the boundary of the range in which the speed characteristic will be measured. Then, the control signal was smoothly changed relative to the equilibrium signal. When the speed exceeds the lower boundary, the control signal is changed in proportion to the actuator position: the control signal is increased from the minimum to the maximum value in the first half of the stroke and decreased in the second half of the stroke. A similar procedure is performed on the reverse actuator stroke. Since dynamic characteristics of the actuator and hardware of the control unit have a significant influence on determination of the speed characteristic by this method, current of input signal must be processed during analysis taking into account the dynamic parameters determined at the previous stages. 


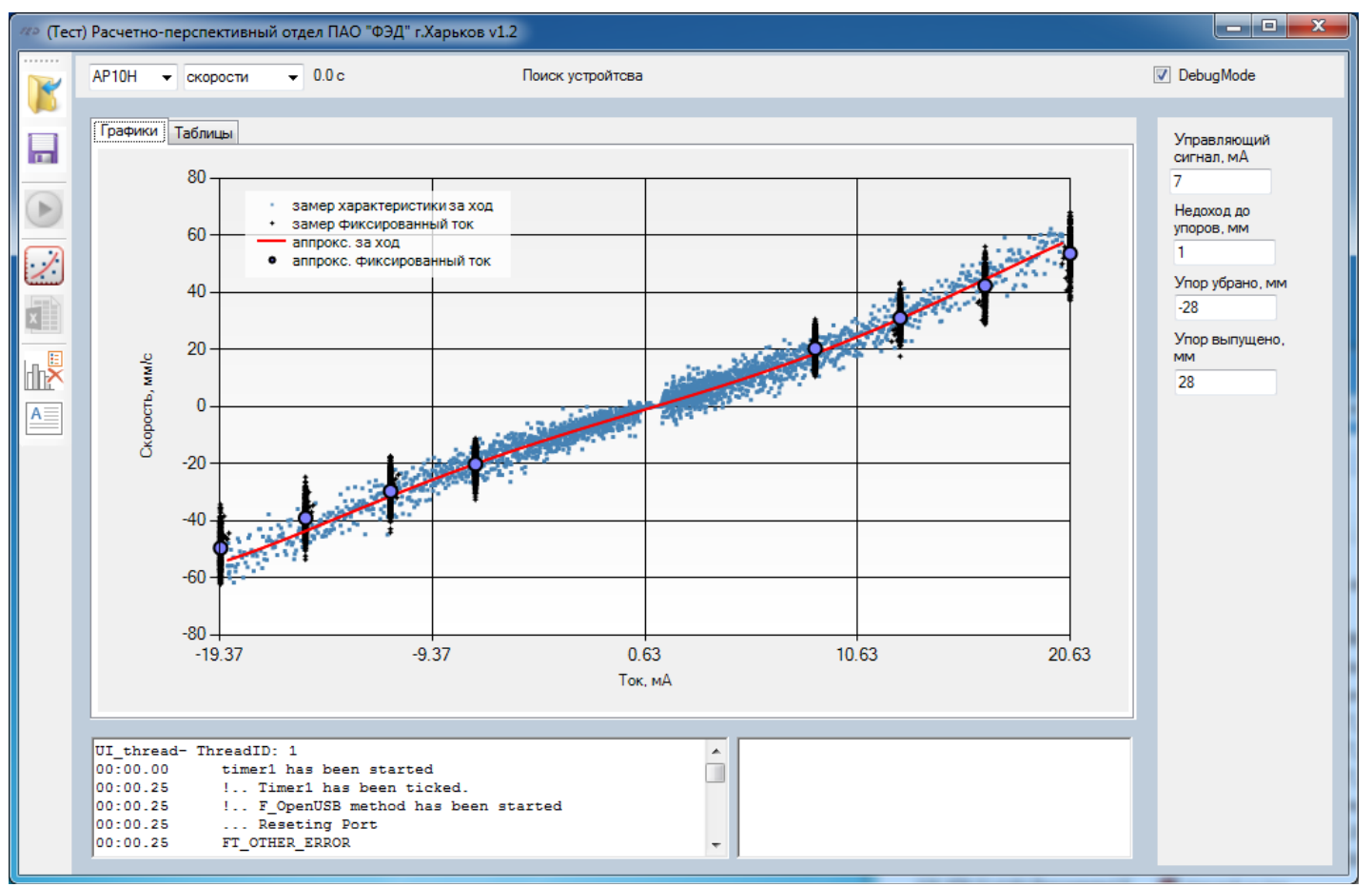

Fig. 5. Software interface when reading speed characteristics

The speed characteristics (Fig. 5) have a strong signal noise associated with a low bit size of the analog-to-digital converter (ADC). To analyze speed characteristics in software, several ways of signal filtering are available: with the use of digital filters having infinite and finite pulse characteristics and by approximating the Bezier curve points (the polynomial degree can be adjusted). The Bezier curve takes the form:

$$
V(I)=\sum_{i=0}^{n} P_{i} \frac{n !}{i !(n-i) !} I^{i}(1-I)^{n-i}
$$

where $P_{i}$ is the function of components of vectors of the datum vertices; $I$ is the control signal; $V$ is the speed of the actuator movement.

During the curve approximation, we searched for the function of components of vectors of the datum vertices using partial derivatives of quadratic deviations of the Bezier curve of the first and the second order by a method similar to approximation by a sinusoidal function described above. In approximation, a strict condition is set for intersection of the speed characteristic of the zero shift point $I_{0}$ which was determined at the previous stage.

To analyze nonlinearity of the speed characteristic, the entire graph of the speed characteristic is approximated by a linear function followed by determination of the speed characteristic nonlinearity. To determine asymmetry in terms of strengthening of the speed characteristic of the actuator, positive and negative portions of the speed characteristic are approximated by individual linear functions.

\section{Discussion of results of studying the methods for determining parameters of a simplified model of an electro-hydraulic actuator}

The proposed methods for determining actuator parameters make it possible to eliminate effects of mutual influence of the actuator characteristics and hardware of the electronic control unit by testing with the use of the standard part of the unit of electronic control of the object on which the actuator is installed. The developed simplified model of the actuator control loop ensures determination of the model parameters with a high stability of results convergence and speed without operator intervention. The model of the electro-hydraulic actuator with hardware of the electronic unit (Fig. 1) has a structure adapted to the procedure of automatic determination of model parameters from experimental data. The following simplifications were adopted in the actuator model:

- the friction model represented by a static force;

- no pure lag in the model structure;

- the model of electro-hydraulic amplifier represented by speed characteristics.

Application of standard detailed friction models [21] used in modern packages is difficult because of a great number of variables used in the model structure:

$$
F_{f r}=\sqrt{2 e}\left(F_{b r k}-F_{C}\right) \exp \left(-\left(\frac{v}{v_{S t}}\right)^{2}\right) \frac{v}{v_{S t}}+F_{C} \tanh \left(\frac{v}{v_{k}}\right)+f v,
$$

where $F_{b r k}$ is the breakaway friction; $F_{C}$ is the Coulomb friction force; $f$ is the viscous friction coefficient; $v$ is the relative velocity; $v_{S t}$ is the Stribek speed threshold; $v_{k}$ is the Coulomb velocity threshold.

Identification of the above detailed model of friction is difficult and inexpedient because of insignificant variation of the friction force in the process of the hydraulic cylinder movement (horizontal section of the graph in Fig. 3).

Absence of a pure delay in the system model (Fig. 1) is caused by a significant worsening of convergence of the sought values of the actuator time constant, hysteresis and pure delay. This effect is caused by similarity of the lag transfer characteristics and combination of the transfer function with hysteresis. The use of an aperiodic link in the model instead of transfer functions of a higher order 
was caused by absence of visible harmonics in the actuator transients (Fig. 4).

When constructing detailed models of the electrohydraulic amplifier operation process, multidisciplinary problems of hydraulics, mechanics, electricity and magnetism are solved. Behavior of hydraulic, electrical and mechanical systems is described using nonlinear nonstationary analytical equations. A similar model of an electrohydraulic amplifier was described in [22]. However, the number of parameters of such models can reach several hundred including geometric parameters of structural elements, material parameters, correction factors, etc. Detailed models of electro-hydraulic amplifier operation process are used primarily for design calculations. They have low speed because of a considerable number of parameters and structural complexity. To construct a fast-computation model, it was proposed to use a mathematical model of an electro-hydraulic converter represented by speed characteristics. In this case, the time constant and hysteresis of the electro-hydraulic converter were combined with parameters of hardware of the electronic control unit.

For automatic identification of the mathematical model of the actuator, procedures for determining the model parameters optimized for the model structure have been developed. Based on these procedures, algorithms and software have been developed for automatic reading and analysis of the actuator characteristics. When developing the electronic part of test benches, two approaches are most widely used at present. The first approach is based on standard data acquisition and processing systems from companies such as National Instruments. These systems are optimized for the structures consisting of standard units of a concrete manufacturer. Each of the standard units is a separate unit for processing or converting information with its own boards, power supply system, etc.

In the second approach, systems based on standard microcontrollers are used. It is necessary to develop electrical circuits for processing and converting signals, supplying circuit elements with power, etc. for each specific design of the test bench.

Upon comparative analysis, the second approach was chosen. The decisive criterion of choosing the second approach was its high flexibility when integrating standard electronic control units of the actuators unit in the hardware system.

The lower-level software embedded in microcontrollers of the electronic control unit of the bench was realized in the $\mathrm{C}++$ programming language.

The higher-level software with a user interface (computer installed) was developed in the $\mathrm{C} \#$ programming language.

The dsPIC series chips for processing signals from the test bench sensors and Cortex-M series chips for controlling the test bench and communicating with the central computer were used as microcontrollers. Hercules Arm Cortex-R microcontroller in which the part of the algorithm responsible for processing and shaping signals was similar to the standard object control unit was used in hardware of the standard control unit. Special boards have been developed for converting analog and discrete signals.

In addition to the algorithms of determining the model parameters, algorithms of automatic determination of main actuator parameters and characteristics (zero shift, dead space, amplitude-frequency, phase-frequency and speed characteristics) have been implemented in the software. The proposed method for automatic determination of the above parameters and characteristics can materially simplify and speed up the process of acceptance tests.

Development of methods for automatic identifying a simplified mathematical model of an electro-hydraulic actuator control loop is a continuation of the author's work in development of electro-hydraulic actuator models. Unlike the detailed workflow model partially described in [22], the simplified model proposed in this study has a simpler structure. Essential simplification of the model ensures automatic identification of various deviations in parameters in various actuator operation conditions (external factors). The ability to analyze the range of change of the actuator model parameters makes it possible to improve quality of synthesis of control algorithms.

A further continuation of studies in development of simplified models of electro-hydraulic actuators may consist in combination of an actuator model with a simplified workload model for various operating conditions.

The proposed procedures can substantially reduce labor costs in processing the experimental data and reduce time of reading experimental characteristics of the actuator.

\section{Conclusions}

1. To determine main dynamic and static characteristics of the electro-hydraulic actuator loop, procedures have been developed for automatic determination of zero shift, dead space, amplitude-frequency, phase-frequency and speed characteristics. These procedures are based on automatic generation of control signals and processing of experimental data without operator participation. The use of experimental data processing procedures adapted to the structure and parameters of electro-hydraulic actuators has increased stability and performance of algorithms. Automation of the characterization process has allowed us to reduce the time taken to read out characteristics from 30 minutes to 1 minute and analysis time from 60 minutes to 8 seconds. In addition, quality and volume of the analyzed data were improved due to exclusion of the human factor and the method for analyzing the actuator speed characteristics and hysteresis with a continuous change of the control signal.

2. A mathematical model of an actuator with a simple structure and high accuracy and methods for automatic determination of its parameters were developed. Simplification of the model was achieved by eliminating the use of workflow models of electrical, magnetic, hydraulic and mechanical components of each of the elements of the control loop separately. It was proposed to use sequential positioning of elements imitating behavior of the entire control loop as a whole: total hysteresis and transfer function, effect of external load and speed characteristic. Structure of the simplified model was chosen in such a way as to ensure accuracy of reproduction of characteristics substantially higher than variation of parameters between actuators with a minimum number of the model elements. Because of simplification, this approach does not enable analysis of influence of parameters of individual structural elements of the control loop on parameters of the entire loop in general. Accuracy of the mathematical model is lower compared to the workflow model. The error in positioning of the output link using the proposed simplified model when working with sinusoidal signals having amplitude of $2 \%$ and $50 \%$ 
of the nominal control signal was not more than $10 \%$ and $3 \%$, respectively, with variation of parameters between the actuators $70 \%$ and $15 \%$, respectively. The error in determining hysteresis was less than $5 \%$ (commensurate with the measurement error) at a spread of parameters among the actuators up to $200 \%$. Thus, the model reproduces basic dynamic and static parameters of the actuator with a distortion an order of magnitude lower than the parameter spread among the actuators. This enables a highly accurate estimate of stability margin of the control loop for each of the produced actuators by means of calculation and simplifies the task of controller synthesis.

\section{References}

1. Erofeev E. V. Sistema rulevyh privodov i energeticheskiy kompleks perspektivnyh samoletov transportnoy kategorii // Elektrifikaciya letatel'nyh apparatov. Tr. nauch.-tekhn. konf. Moskva: ID Akad. Zhukovskogo, 2016. P. 178-187.

2. New developments of electrically powered electrohydraulic and electromechanical actuators for the more electric aircraft / Ogoltsov I., Samsonovich S., Selivanov A., Alekseenkov A. // 29th Congress of the International Council of the Aeronautical Sciences. Saint Petersburg, 2014.

3. Test Set-up for Electromechanical Actuation Systems for Aircraft Flight Control / Barnett S. A., Lammers Z., Razidlo B., Leland Q., DelMar J. // SAE Technical Paper Series. 2012. doi: https://doi.org/10.4271/2012-01-2203

4. R50-109-89. Rekomendacii. Nadezhnost' v tekhnike. Obespechenie nadezhnosti izdeliy. Obshchie trebovaniya. Moscow, 1989.

5. Actuators: Aircraft Flight Controls, Power Operated, Hydraulic, General Specification For. ARP 1281. SAE. doi: https://doi.org/ $10.4271 / \operatorname{arp} 1281$

6. Acceptance test for the linear motion actuator for the scanning slit of the hie-isolde short diagnostic boxes / Cantero E. D., Andreazza W., Bravin E., Sosa A. // European organization for nuclear research. CERN-ACC-NOTE-2014-0099. HIE-ISOLDEPROJECT-Note-0036. Geneva, 2014.

7. Gilson E., Kopp J. D., Manzanares D. Moog next generation control and actuation // R3ASC. Toulouse, 2014. P. $43-54$.

8. Chan C.-H., Liu G. Actuator hysteresis identification and compensation using an adaptive search space based genetic algorithm // Proceedings of the 2004 American Control Conference. 2004. doi: https://doi.org/10.23919/acc.2004.1384775

9. A diagnostic approach for electro-mechanical actuators in aerospace systems / Balaban E., Bansal P., Stoelting P., Saxena A., Goebel K. F., Curran S. // IEEE Aerospace conference. 2009. doi: https://doi.org/10.1109/aero.2009.4839661

10. Combining model-based and feature-driven diagnosis approaches - a case study on electromechanical actuators / Narasiman S., Roychoudhury I., Balaban E., Saxena A. // 21st International workshop on principles of diagnosis. 2010.

11. Langjord H., Kaasa G.-O., Johansen T. A. Nonlinear observer and parameter estimation for electropneumatic clutch actuator. URL: http://folk.ntnu.no/torarnj/Nolcosver2.pdf

12. Desborough L., Miller R. Increasing customer value of industrial control performance monitoring -honeywell's experience // Chemical Process Control. 2001. P. 172-192.

13. Choudhury A. S., Shah S. L., Thornhill N. F. Diagnosis of Process Nonlinearities and Valve Stiction. Data Driven Approaches. Springer, 2008. 286 p. doi: https://doi.org/10.1007/978-3-540-79224-6

14. Liang L., Jiannan L., Wan H. Parameter estimation for linear control valve with hysteresis // Submitted to IEEE transactions on automation science and engineering. 2014. URL: https://arxiv.org/pdf/1605.00347.pdf

15. Shi W. Electro-Hydraulic Servo-Valve and Motion and Control Loading of Full Flight Simulator. URL: https://digital.library.ryerson.ca/islandora/object/RULA\%3A2203/datastream/OBJ/download/Electro-Hydraulic_Servo-Valve_and_Motion_and_Control_Loading_of_Full_Flight_Simulator.pdf

16. Xu Y. Modelling and control of a high performance electro-hydraulic test bench. INSA de Lyon, 2013.

17. Michel R. A simple method to determine control valve performance and its impacts on control loop performance. URL: http://www. topcontrol.com/fichiers/en/controlvalveperformance.pdf

18. Galinaitis W. S., Joseph D. S., Rogers R. C. Parameter Identification for Preisach Models of Hysteresis // ASME Design Engineering Technical Conferences. Pittsburgh, 2001.

19. Abbasov M. E. Metody optimizacii: ucheb. pos. Sankt-Peterburg, 2014.

20. Grigor'eva K. V. Metody resheniya zadachi minimizacii kvadratichnoy funkcii: ucheb. pos. Sankt-Peterburg, 2007.

21. Armstrong B., Wit C. C. Friction Modeling and Compensation. The Control Handbook. CRC Press, 1995.

22. Kononykhin E. A., Yepifanov S. V. Backfitting of servovalve characteristics using unit level dynamic models // Aviacionno-kosmicheskaya tekhnika i tekhnologiya. 2016. Issue 6. P. 48-54. 